\title{
Os Determinantes Regionais da Atração do Migrante Qualificado
}

\section{The Regional Determinants of Skilled Migrants Attraction}

\author{
Juliana Gonçalves Taveira* \\ Eduardo Almeida**
}

\begin{abstract}
Resumo: A mobilidade de trabalhadores, em especial dos qualificados, se destaca na literatura como uma das principais formas de difusão de conhecimento. Sendo assim, a partir de um painel balanceado composto por 5.015 municípios e abrangendo os anos de 2001 e 2007, o presente estudo visa a avaliar as características das cidades que mais atraem o migrante qualificado. Constata-se que o modelo sem controle espacial fornece estimativas viesadas, enfatizando, assim, a importância do controle da dependência espacial e das características regionais não observadas na estimação do modelo. Ao controlar para dependência espacial, observa-se uma preferência dos migrantes qualificados por cidades com renda esperada, população, PIB per capita e grau de industrialização elevadas. Além disso, preferem tanto cidades com menor criminalidade e tráfego, quanto cidades que oferecem melhor transporte público. Os principais resultados revelam a relevância das características regionais dos vizinhos para explicar os fluxos migratórios de pessoas qualificadas.
\end{abstract}

Palavras-chave: Migração de qualificados. Efeitos não observados. Dependência espacial.

\begin{abstract}
Workers' mobility, particularly the skilled ones, appears in the literature as one of the major forms of knowledge spillovers. Thus, using a balanced panel of 5015 municipalities over the years 2001 and 2007, this study is aimed at appraising what cities' characteristics attract skilled migrants. The model with no spatial control provides biased estimates. This sheds lights on the importance of controlling the spatial dependence and unobserved regional characteristics in the estimation of the model. After controlling for spatial dependence, there is evidence that skilled migrants prefer cities with higher expected income, population, GDP per capita and degree of industrialization. Furthermore, they prefer both cities with less crime and traffic and cities that provide better public transportation. The main findings reveal the relevance of surrounding regional characteristics to explain skilled migration flows.
\end{abstract}

Keywords: Skilled workers migration. Non-observed effects. Spatial dependence.

JEL Classification: J61; O1; R10; R23.

\footnotetext{
* Doutoranda em Economia Aplicada pelo Programa de Pós-Graduação em Economia da Universidade Federal de Juiz de Fora (UFJF). Professora assistente do Departamento de Economia da UFJF. E-mail: julianagtaveira@gmail.com

** Professor associado do Departamento de Economia da UFJF. Pesquisador do CNPq e da Fundação de Amparo à Pesquisa do Estado de Minas Gerais (Fapemig).E-mail: eduardo.almeida@ufff. edu.br

Os autores agradecem o suporte financeiro do CNPq, da Fapemig e da UFJF.
} 


\section{Introdução}

A mobilidade de trabalhadores, em especial dos qualificados, constitui uma das principais formas de difusão de conhecimento tecnológico (MUKKALA, 2005), uma vez que tais profissionais transportam o conhecimento tácito, um tipo de conhecimento que só pode ser transmitido via contato direto. Altos níveis de mobilidade de pessoas qualificadas asseguram o nível de inovação e de desenvolvimento econômico da região (GRAVERSEN; FRIIS-JENSEN, 2001). Por intermédio da migração, portanto, regiões terão acesso a conhecimentos que, por outro meio, não teriam.

De acordo com a literatura, o migrante é autosselecionado, ou seja, apresenta-se mais motivado e habilidoso que os outros indivíduos (BORJAS, 1987). No Brasil, as migrações internas se destacam no processo de desconcentração e redistribuição populacional ao criar novos núcleos econômicos e desenvolver as cidades (MATOS; BRAGA, 2002).

Golgher, Rosa e Araújo Júnior (2005) afirmam ainda que as migrações internas estão altamente associadas ao desenvolvimento econômico das regiões. Além disso, destacam que indivíduos com baixa renda, ao migrar, levarão em conta principalmente variáveis econômicas. Já os com renda superior visarão às características do destino e variáveis não econômicas.

Até a década de 1970, o processo migratório e a economia brasileira envolviam principalmente a região sudeste, e em especial a cidade de São Paulo. Contudo, a partir daí, ocorreu uma reversão desse processo (QUEIROZ; CÉSAR, 2000). Baeninger (2005) comprova o processo de desconcentração da migração do estado de São Paulo e destaca uma expansão das áreas de recepção dos migrantes no interior.

As migrações internas no Brasil passam a se destacar no processo de desconcentração e redistribuição populacional, uma vez que criam novos núcleos econômicos e desenvolvem as cidades (MATOS; BRAGA, 2002). O movimento migratório da metrópole em direção ao interior intensifica a dispersão populacional (BAENINGER, 2005).

A migração brasileira está diretamente associada à evolução das características regionais, como desigualdade de renda per capita e densidade populacional, e aos aspectos históricos da distribuição populacional (GOLGHER; ROSA; ARAÚJO JÚNIOR, 2005). Apesar de o principal destino dos migrantes ainda ser as regiões metropolitanas, eles estariam deixando de se referenciar nos centros urbanos tradicionais, uma vez que o dinamismo do mercado de trabalho das regiões afastadas das grandes aglomerações os estaria atraindo. Ademais, estariam mudando, além de região, de setor (FERREIRA; MATOS, 2006). 
No Brasil, segundo Rigotti (2006), a maior parte dos migrantes educados tinha, em sua origem, uma região metropolitana ou uma capital. Tais regiões se mostram importantes à medida que possuem um grande número de universidades e de centros de pesquisa, sendo assim fornecedores de mão de obra qualificada. Queiroz e Golgher (2008) apontam, ainda, que os trabalhadores qualificados tendem a se dirigir para cidades com mais centros educacionais, o que pode acarretar uma concentração desses indivíduos nas regiões mais desenvolvidas. Além disso, esses trabalhadores estariam distribuídos de forma polarizada ao redor ou dentro das áreas metropolitanas (GOLGHER, 2008).

Os movimentos migratórios brasileiros se caracterizam por uma emigração dos estados com renda per capita menor para as regiões com renda per capita maior, assim estas estariam concentrando indivíduos mais hábeis e, portanto, mais produtivos (SANTOS JÚNIOR; MENEZES FILHO; FERREIRA, 2005). Menezes e Ferreira Júnior (2003) destacam que as cidades grandes se caracterizam como principal destino dos migrantes devido a sua renda esperada e sua densidade demográfica serem mais elevadas.

Na teoria do crescimento descrita por Romer (1990), destaca-se a relação entre crescimento e acumulação e difusão de conhecimento como fundamento para o crescimento econômico endógeno. Existem vários meios pelos quais o conhecimento pode se difundir através das regiões, mas o tácito é principalmente transmitido por meio do contato interpessoal. Logo, a mobilidade de trabalhadores qualificados possui consequências importantes no desenvolvimento regional. Além disso, aponta-se ainda que o nível educacional dos migrantes é significativamente maior se comparado com os não migrantes (STAMBOL, 2003), o que comprova a importância de se investigar a mobilidade dos trabalhadores.

A maioria dos estudos a respeito da mobilidade de trabalhadores busca investigar os fatores individuais que determinam o fenômeno, sendo que dos trabalhos com enfoque nas características regionais que caracterizam a migração, a sua maioria não controla para efeitos não observados e/ou dependência espacial, o que poderia viesar os resultados encontrados. Além disso, não se encontraram na literatura especializada trabalhos analisando a influência da vizinhança nos fluxos migratórios.

Assim, visando a preencher essa lacuna existente, o presente artigo utiliza variáveis referentes ao destino para determinar quais características municipais mais incentivam os migrantes qualificados a se dirigirem para a região. Para isso, utiliza-se um painel balanceado composto por 5.015 municípios, compreendendo os anos de 2001 e 2007.

O trabalho busca compreender quais fatores de uma região atraem fluxos migratórios de trabalhadores qualificados, dado o papel desses indivíduos no desenvolvimento econômico e social de uma região. Assim, dada a importância do 
controle da dependência espacial e das características não observadas na estimação dos modelos em nível regional, o artigo inova ao estimá-lo por meio de um painel de dados, controlando-se para dependência espacial.

Sendo assim, constata-se que o modelo sem controle espacial fornece estimativas viesadas. Ao controlar para dependência espacial, observa-se uma preferência dos migrantes por cidades com renda esperada, população, PIB per capita e grau de industrialização elevadas. Além disso, observa-se que os migrantes priorizam cidades com menor criminalidade e tráfego e melhor transporte público. Por fim, pode-se concluir que o município concorre com seus vizinhos à medida que cidades com maiores proporção de migrantes qualificados estão rodeadas por municípios com baixa entrada de migrantes e de renda esperada.

O presente trabalho está organizado em cinco seções: esta primeira introduz a temática estudada; a segunda faz uma revisão da literatura nacional e internacional sobre o tema; a terceira apresenta a metodologia utilizada e descreve a base de dados; a quarta discute os resultados encontrados; e, por fim, as considerações finais recupera os principais pontos e conclusões do trabalho.

\section{Revisão de Literatura}

\subsection{Determinantes e Características da Migração}

O capital humano é fator chave no desenvolvimento regional, sendo que a maioria dos estudos é unânime em concluir que a propagação de conhecimento é relevante. A migração se destaca como importante meio de difusão do conhecimento entre as regiões, podendo o fenômeno levar a mudanças significativas nos estoques regionais de capital humano (MUKKALA, 2005). Assim, regiões terão acesso a conhecimentos que as inserirão nos sistemas de inovação e que, por outro meio, não ingressariam.

Os movimentos migratórios se tornam um dos mecanismos para amenizar as diferenças estruturais nos mercados de trabalho entre as regiões, como diferenças de taxas de desemprego e de aglomerações de capital humano (JACKMAN; SAVOURI, 1992; DEBELLE; VICKERY, 1999). Além disso, a mobilidade geográfica dos indivíduos é também um mecanismo importante de alocação eficiente de mão de obra (JAEGER et al., 2007).

A migração muda as características regionais tanto em termos de taxa de crescimento populacional quanto em termos de composição populacional e distribuição de capital físico e humano. As consequências da migração para as regiões são ambíguas, mas regiões que atraem uma grande proporção de migrantes possuem uma maior proporção de capital humano qualificado (GOLGHER; ROSA; ARAÚJO JÚNIOR, 2005). 
Dependendo das características regionais e dos tipos de migrantes que cada região atrai, ou perde, ela se beneficiará, ou não, da migração (GOLGHER, 2008). A mobilidade geográfica de trabalhadores é vista como um mecanismo de ajuste de diferenças regionais de capital humano e de crescimento econômico (ZIMMERMANN, 2005).

Sachsida, Caetano e Albuquerque (2010) apontam que, à medida que os mais aptos de uma localidade se dirigem a outra, a oferta de trabalho desses trabalhadores aumenta, mas, se estes forem menos aptos do que os da região de destino, o aumento da oferta de trabalho será de indivíduos menos qualificados. Se houver substitutibilidade entre os qualificados e os não qualificados, a demanda pelos primeiros cai, mas, se houver complementaridade, esta procura aumenta e a desigualdade de renda cresce. Golgher, Rosa e Araújo Júnior (2005) apontam ainda que regiões que atraem um grande número de migrantes tendem a ter sua população jovem maior que as outras regiões.

Níveis significantes de mobilidade de pessoas qualificadas ou de trabalhadores com nível alto de inovação asseguram o grau de atividades inovadoras das regiões e garantem o desenvolvimento. A migração desses trabalhadores possibilita a mobilidade do conhecimento, garantindo a difusão e circulação do conhecimento intrínseco ao indivíduo, a saber, do conhecimento tácito (GRAVERSEN; FRIIS-JENSEN, 2001). Ao considerar que a mão de obra qualificada não migra entre firmas e/ou regiões, algumas destas poderiam ter seu crescimento econômico comprometido (DAHL, 2004).

Migrantes são considerados autosselecionados positivamente em relação aos indivíduos que optam por permanecer em seu lugar de origem, ou seja, são em média mais ambiciosos, empreendedores e habilidosos (CHISWICK, 1999). Freguglia e Menezes Júnior (2007) apontam a importância das habilidades individuais não observadas como fator determinante dos diferenciais salariais no mercado de trabalho formal entre os estados brasileiros. Infere-se, portanto, a importância da inclusão desse fator não observado ao analisar os impactos e os determinantes da migração para o melhor entendimento do processo.

Essa habilidade dos migrantes pode ajudar a explicar um crescimento superior das regiões onde ocorre uma concentração de migrantes qualificados e o fato de o salário do migrante ser maior em relação aos nativos. Santos Júnior, Menezes Júnior e Ferreira (2005) descobrem que o migrante brasileiro é selecionado positivamente e destacam ainda que como este se dirige principalmente para as regiões mais ricas, esse movimento estaria piorando a desigualdade de renda entre as regiões brasileiras.

A migração deve ser entendida como um investimento em capital humano feito pelo indivíduo. Ao migrar, inicialmente, ele incorre em gastos, monetários e não monetários, e almejará um retorno do investimento com o decorrer do tem- 
po (SJAASTAD, 1962). Com sua decisão de migrar, o migrante busca maximizar lucros e minimizar custos. Sendo assim, a migração será uma busca por um maior nível de bem-estar (SACHSIDA; CAETANO; ALBUQUERQUE, 2010).

A decisão de migrar, portanto, ocorrerá apenas quando houver chances reais de se recuperar esses investimentos, assim como é determinada por características do indivíduo e das cidades de destino e de origem. Aqueles fatores que aumentarem as chances de retorno do investimento terão relação positiva, e os que dificultarem terão relação negativa com a migração.

Uma estrutura teórica presente na literatura que destaca a migração de indivíduos como uma resposta a características econômicas, sociais e políticas das regiões é denominada push-pull theory (teoria do empurra-puxa). Os fatores push seriam as características da região de origem que repelem o migrante e pull aquelas da de destino que o atraem (MATA et al., 2008). Normalmente, pessoas com uma renda inferior seriam mais influenciadas pelos fatores push e os com renda superior pelo pull (GOLGHER; ROSA; ARAÚJO JÚNIOR, 2005).

A migração depende do salário per capita, taxa de desemprego, nível de pobreza da região e provisão de bens públicos (ANDRIENKO; GURIEV, 2004). A decisão de migração responde significativamente a diferenças regionais de riqueza, salários, taxas de desemprego e custo de vida (ZIMMERMANN, 2005). Evidências empíricas comprovam uma correlação entre a probabilidade de migração e os diferenciais de renda entre as regiões e uma relação negativa dessa probabilidade com a distância entre as regiões de destino e origem (BORJAS, 1996).

De acordo com Andrienko e Guriev (2004), a migração depende de fatores econômicos, uma vez que as pessoas se movem de regiões pobres, com altas taxas de desemprego e com pior provisão de bens públicos, para cidades com melhores condições. Entretanto, ao mesmo tempo em que um nível salarial mais baixo incentiva a saída de trabalhadores, impede que estes tenham condições financeiras de fazê-lo.

Se a diferença de nível de renda entre as regiões for pequena, apenas uma pequena parcela da população estará disposta a migrar. Tal porcentagem aumenta à medida que a diferença superar o investimento inicial incorrido com a migração (ANDRIENKO; GURIEV, 2004). A decisão de migrar está correlacionada com as características do mercado de trabalho (DEBELLE; VICKERY, 1999). Estudos da Suécia (NAKOSTEEN; WESTERLUND, 2004), do Reino Unido (JACKMAN; SAVOURI, 1992), da Espanha (BOVER; ARELLANO, 2002) e da Austrália (DEBELLE; VICKERY, 1999) comprovam uma relação positiva entre condições favoráveis de mercado e a decisão de migrar.

A taxa líquida de imigração estadual nos Estados Unidos, conforme destacado por Cebula e Alexander (2006), é função crescente da renda média familiar e da taxa de crescimento do desemprego do período anterior e decrescente do 
custo de vida. Já Mata et al. (2007) apontam que, no Brasil, observa-se uma maior procura por parte dos migrantes qualificados de cidades com maiores salários. Além disso, destaca-se que a renda esperada é importante para explicar a taxa líquida de migração (JUSTO; SILVEIRA NETO, 2006).

Menezes e Ferreira Júnior (2003) constatam, no Brasil, uma relação positiva entre a renda do trabalhador ao tomar a decisão de migrar e a densidade demográfica dos estados com a taxa líquida de migração. Os autores apontam ainda que tal relação indicaria que os fluxos migratórios estão em consonância com a teoria de aglomeração, ou seja, se dirigem às grandes cidades onde a renda esperada e a densidade demográfica são maiores.

O desemprego entre as regiões se mostra como fator determinante da migração, afetando-a de três formas: primeiramente, a partir do trabalhador, que ao estar desempregado possui maior probabilidade de migrar por não ter que ser compensado por abrir mão do emprego na origem; segundo, o nível regional do desemprego na origem determina a necessidade de migrar em busca de cidades com maior número de emprego ou não; e terceiro, se a taxa de desemprego geral for alta, o valor do emprego aumenta e os trabalhadores empregados tenderão a migrar menos. Apesar da alta relação entre desemprego e migração, os diferenciais regionais de desemprego não influenciam a migração de forma independente (PISSARIDES; WADSWORTH, 1989).

Cebula (2005) aponta que os diferenciais das taxas de desemprego não influenciam a imigração. Contudo, destaca que a imigração tem relação positiva com a renda per capita esperada e renda per capita real e relação negativa com a média do custo de vida. Os indivíduos migram principalmente de regiões com baixa renda per capita para lugares com maiores salários e melhores oportunidades de emprego. Dessa forma, indivíduos com renda menor, ao tomar a decisão de migrar, darão maior importância a variáveis econômicas, e, os com renda maior, às características do destino e a aspectos não econômicos (GOLGHER; ROSA; ARAÚJO JÚNIOR, 2005).

Uma das principais características do destino a ser considerado na hora de tomar a decisão de migrar é o custo de vida. Jackman e Savouri (1992) constataram, no Reino Unido, que o custo de vida influencia positivamente a emigração e, negativamente, a imigração. Os autores apontam ainda que a existência de casa própria na cidade de origem seria um fator que influenciaria negativamente a decisão de migrar. Tal fenômeno também foi constatado na Espanha, onde as pessoas se dirigiram para as cidades pequenas e médias, pois o custo de vida é menor (BOVER; ARELLANO, 2002).

Em estudo sobre o Brasil, Oliveira e Jannuzi (2005) verificaram que as pessoas residentes ou que saíram de regiões metropolitanas tendem a ter como principal motivação da migração o custo de vida. Já as migrações de retorno dos 
nordestinos ocorreriam devido a dificuldades de inserção no mercado de trabalho e à violência na região de destino.

O tamanho da população tanto da origem quanto do destino é determinante da migração (SACHSIDA; CAETANO; ALBUQUERQUE, 2010). Um maior número de ônibus, hospitais, densidade rodoviária e telefone per capita diminui o número de fluxos emigratórios, ao mesmo tempo em que estimula a imigração. As regiões com uma grande densidade populacional enviam e recebem um número maior de migrantes (ANDRIENKO; GURIEV, 2004). Mata et al. (2007) destacam que, para os migrantes qualificados brasileiros, a qualidade do sistema de saúde não é importante ao decidir para onde migrar.

Os autores apontam que os migrantes qualificados são atraídos por cidades com menor instabilidade social, assim se dirigem principalmente para regiões com menores níveis de desigualdade e de violência. Entretanto, ainda para o Brasil, Sachsida, Caetano e Albuquerque (2010) descobrem que a violência em relação aos fluxos migratórios se mostrou estatisticamente não significativa. Já Golgher, Rosa e Araújo Júnior (2005) destacam que a taxa de crime afeta a migração intraurbana, apesar de não interferir na migração inter-regional. Justo e Silveira Neto (2006) encontram, por sua vez, que o nível de criminalidade tem relação negativa com a taxa líquida de migração.

Apesar da preferência por regiões onde os diferenciais de renda sejam menores, os migrantes amantes do risco tendem a se dirigir para locais com maior desigualdade, pois visam a um ganho pós-migração maior, mesmo o risco também sendo superior (SACHSIDA; CAETANO; ALBUQUERQUE, 2010).

Sachsida, Caetano e Albuquerque (2010) afirmam que as transferências federais têm relação com o fluxo imigratório, predominando a ida de migrantes para regiões que recebem maiores transferências e possuem um nível de renda e população maiores. A concentração de renda, todavia, teria correlação negativa com a imigração, o que também evidencia que os migrantes têm preferência por cidades com a distribuição de renda mais equitativa.

A imigração líquida estadual é função crescente dos gastos do governo local por aluno sobre a educação primária e secundária e uma função decrescente da carga fiscal sobre o rendimento individual do estado. Além disso, os encargos do imposto de renda determinam parte da imigração líquida (CEBULA; ALEXANDER, 2006).

Cabe destacar que a capacidade de atração de migrantes por uma região está intimamente relacionada aos seus vizinhos (JUSTO; SILVEIRA NETO, 2006). Mata et al. (2007) apontam que a recepção de um grande número de migrantes qualificados por parte de municípios vizinhos reduz a absorção do fluxo migratório do município em questão. 
Além disso, a proximidade ao litoral e invernos e verões menos rigorosos são fatores importantes na escolha do destino dos trabalhadores qualificados do Brasil (MATA et al. 2007). Apesar de Mata et al. (2008) destacarem a preferência de migrantes qualificados por regiões com menor desigualdade social, Justo e Silveira (2006), ainda que tenham confirmaram as relações anteriores, apontam a não significância do índice de Gini, medida de desigualdade de renda, em relação à taxa líquida de migração.

Andrienko e Guriev (2004) também apontam que os migrantes russos tendem a migrar para regiões litorâneas e com rios largos, e afirmam, ainda, que regiões com portos atraem de $15 \%$ a $20 \%$ a mais de migrantes. Tal fato estaria correlacionado com maiores oportunidades de empreendedorismo. Para os Estados Unidos, encontra-se que a imigração líquida apresenta relação positiva com temperaturas mais amenas e relação negativa com a poluição. Contudo, as variáveis proxies de proximidade do litoral não se mostraram significativas (CEBULA; ALEXANDER, 2006). A taxa bruta de imigração no país é função crescente da disponibilidade de parques, opções de divertimento e temperaturas mais quentes, e função decrescente de taxa de crime e poluição (CEBULA, 2005).

Chen e Rosenthal (2008) destacam que famílias migrantes tendem a preferir regiões não metropolitanas e áreas em região costeira com temperatura quente. Já os aposentados se dirigem mais para as cidades com maiores amenidades e baixo custo de vida. As famílias altamente educadas, entre 20 e 35 anos, independente do estado civil, se movem para locais com ambiente corporativo desenvolvido. Já os casais com mais de 50 anos, independente do nível educacional, saem de ambiente corporativos e procuram lugares com maiores amenidades.

Jensen e Deller (2007) apontam que a taxa de criminalidade possui relação positiva com a imigração e emigração de idosos, enquanto que uma alta concentração de médicos está associada com níveis maiores de emigração e menores de imigração. Enquanto que cidades com temperaturas mais elevadas aumentam a emigração, o tamanho da superfície d'água detém a saída desses indivíduos. Além disso, os idosos parecem interessados em áreas de lazer.

Fatores ligados à idade do indivíduo, existência de filhos, de esposa ou marido ou relação com a família também influenciam na mobilidade (DAHL, 2004). No caso da Estônia, Kulu e Billari (2004) evidenciam que a probabilidade de migração dos indivíduos varia de acordo com fatores pessoais e regionais. Os autores ratificam a importância da renda, desejos e crenças dos indivíduos no seu comportamento migratório.

É destacado ainda na literatura que idade e nível educacional são as características individuais que mais influenciam na decisão de migração, tendo a primeira correlação negativa e, a segunda, positiva (NAKOSTEEN; WESTERLUND, 2004; KULU; BILLARI, 2004; STAMBOL, 2003; PEKKALA, 2003; SACHSIDA et al. 2009). 
À luz da revisão literária apresentada, observa-se a pouca atenção dada pela literatura empírica pesquisada a cerca do tema para o controle dos efeitos não observados das regiões, assim como do controle da dependência espacial. Adicionalmente, não foram encontrados trabalhos analisando as relações entre os vizinhos na atração dos indivíduos qualificados. Assim, o presente estudo visa a preencher essa lacuna, além de apresentar novas evidências sobre o tema para o Brasil.

\section{Metodologia}

\subsection{Base de Dados e Descrição das Variáveis}

A fim de se observar quais fatores atraem os indivíduos qualificados, ou seja, com nível de escolaridade superior completo para as cidades, utilizou-se um painel balanceado de 5.015 municípios para o período de 2001 e 2007. Adotou-se esta janela temporal, uma vez que um intervalo inferior poderia não ser suficiente para captar mudanças nas características dos municípios.

Assim, usando dados da Relação Anual de Informações Sociais (Rais-Migra), define-se como variável dependente $\left(M Q P_{i}^{t}\right)$ do modelo, descrita na equação 1, a proporção de trabalhadores imigrantes qualificados, ou seja, com nível educacional superior completo, da cidade $i$ no período $t, I Q_{i}^{t}$, sobre o total de trabalhadores com nível superior completo na cidade $j$ no período $t, T Q_{j}^{t}$ :

$$
M Q P_{i}^{t}=I Q_{i}^{t} / T Q_{j}^{t} \quad \text { com } i \neq j
$$

em que $i$ e $j$ variam de 1 a 5.015 e $t$ de 1 a 2 .

Essa fonte de dados permite observar os trabalhadores qualificados do setor formal de trabalho que mudaram de município entre os anos de 2000 e 2001 e 2006 e 2007. Dessa forma, por seu caráter longitudinal, pode-se observar origem e destino dos trabalhadores qualificados migrantes e construir uma medida de diferencial de renda (DR). Esta variável é calculada tirando-se a média da diferença entre a renda média do município de destino observada na origem e a renda que o trabalhador possuía na origem, controlando-se pela educação e por município de origem e destino. Assim, obteve-se a média do diferencial de renda esperado pelo trabalhador. A fim de se manter uma padronização nas unidades de medida, e dado que essa variável era medida em valor nominal, dividiu-se por 1.000.

No caso em que a renda do indivíduo é alta, Golgher, Rosa e Araújo Júnior (2005) destacam que ele, ao migrar, dá maior importância às características do destino e aos aspectos não econômicos. Assim, dado o objetivo do trabalho, e como os trabalhadores qualificados em geral são os com maiores salários, é utili- 
zada a definição dos fatores de atração (pull) para o caso do migrante qualificado, levantada por Mata et al. (2008).

A partir daí obteve-se junto do IBGE os dados de população e PIB municipal para a construção das variáveis de grau de desenvolvimento e tamanho das cidades, sendo utilizadas como proxies as variáveis denominadas POP e PPC, que representam respectivamente o total da população, o PIB per capita. Pela mesma razão apresentada anteriormente para a variável de diferencial de renda, dividiu-se o total da população por 1.000. Além disso, construiu-se, com os dados da Rais o grau de industrialização (GIND) dos municípios, sendo este considerado como a razão entre o total de trabalhadores da indústria de transformação e o total de trabalhadores da economia.

A fim de se medir grau de congestionamento e qualidade do transporte público, utilizou-se, com a base de dados do Denatran, o número de veículos automotores e motocicletas per capita (FROP) e o número de ônibus e micro-ônibus per capita (BUSP). Como medida de violência urbana, calculou-se o número de homicídios, compilado no Datasus, sobre o total da população.

Visando a testar como as características do emprego formal, da saúde e da educação dos municípios afetam a migração, usou-se a decomposição do Índice Firjan de Desenvolvimento Municipal (IFDM) de 2000 como proxy para as características de 2001 e 2007. Optou-se pela escolha desse índice por seu recorte municipal e abrangência nacional e por fazer uso apenas de estatísticas oficiais.

O IFDM é decomposto em emprego e renda (IER), ${ }^{1}$ saúde (ISAU) ${ }^{2}$ e educação (IEDU). ${ }^{3}$ Esses componentes variam de zero (0) a um (1), sendo que, quanto mais próximo de um, maior será o nível de desenvolvimento do município nessas áreas. O IFDM-Emprego e Renda acompanha a movimentação e as peculiaridades do mercado de trabalho formal. Já o IFDM-Educação visa a captar a oferta e a qualidade da educação do ensino fundamental e pré-escola, oferecida nos municípios brasileiros, em escolas públicas e privadas. O IFDM-Saúde avalia a qualidade do sistema de saúde municipal referente à atenção básica.

A fim de se verificar a existência de multicolinearidade construiu-se uma matriz de correlação para as variáveis do modelo. Observa-se que a única correlação acima de 0,8 é encontrada entre a variável FROP e a BUSP, o que já era de se esperar, pois ambas podem ser uma medida para tráfego urbano. No entanto, mantiveram-se as duas, uma vez que com a primeira variável objetiva-se captar congestionamento urbano e com a segunda procura-se capturar a influência do

1 É formado pelas variáveis geração, estoque e salários médios do emprego formal.

2 É composto pelo número de consultas pré-natal, óbitos infantis por causas evitáveis e óbitos por causa mal definida.

3 É formada por taxa de matrícula na educação infantil, taxa de abandono, taxa de distorção idade-série, percentual de docentes com ensino superior, média de horas-aula diárias e resultado do Ideb. 
transporte público. Cabe ressaltar ainda que a variável de diferencial de renda é a que possui maior correlação com a variável dependente. As variáveis, dependente e explicativas estão detalhadas no Quadro 1.

Quadro 1 - Descrição das variáveis utilizadas no modelo

\begin{tabular}{|c|c|c|c|c|c|}
\hline & Variável & Descrição & Unidade & $\begin{array}{l}\text { Sinal es- } \\
\text { perado do } \\
\text { coeficiente }\end{array}$ & \begin{tabular}{|l} 
Fonte \\
dos \\
dados
\end{tabular} \\
\hline $\begin{array}{l}\text { Variável } \\
\text { dependente }\end{array}$ & $\mathrm{MQP}$ & $\begin{array}{l}\text { Trabalhadores } \\
\text { imigrantes qua- } \\
\text { lificados sobre } \\
\text { a população }\end{array}$ & Proporção & ------ & $\begin{array}{l}\text { Rais- } \\
\text { Migra }\end{array}$ \\
\hline \multirow{10}{*}{$\begin{array}{l}\text { Variáveis } \\
\text { explicativas }\end{array}$} & $\mathrm{DR}$ & $\begin{array}{l}\text { Diferencial de } \\
\text { renda }\end{array}$ & Proporção & Positivo & $\begin{array}{l}\text { Rais- } \\
\text { Migra }\end{array}$ \\
\hline & POP & $\begin{array}{l}\text { Total da popu- } \\
\text { lação }\end{array}$ & Proporção & Positivo & IBGE \\
\hline & GIND & $\begin{array}{l}\text { Grau de indus- } \\
\text { trialização }\end{array}$ & Proporção & Positivo & Rais \\
\hline & ISAU & $\begin{array}{l}\text { Índice Firjan } \\
\text { de Saúde }\end{array}$ & Índice & Positivo & Firjan \\
\hline & IER & $\begin{array}{l}\text { Índice Firjan } \\
\text { de emprego e } \\
\text { renda }\end{array}$ & Índice & Positivo & Firjan \\
\hline & IEDU & $\begin{array}{l}\text { Índice Firjan } \\
\text { de educação }\end{array}$ & Índice & Positivo & Firjan \\
\hline & HOMP & $\begin{array}{l}\text { Número de } \\
\text { homicídios } \\
\text { sobre a popu- } \\
\text { lação }\end{array}$ & Proporção & Negativo & Datasus \\
\hline & FROP & $\begin{array}{l}\text { Número de } \\
\text { veículos sobre } \\
\text { a população }\end{array}$ & Proporção & Negativo & Denatran \\
\hline & BUSP & $\begin{array}{l}\text { Número de } \\
\text { ônibus sobre a } \\
\text { população }\end{array}$ & Proporção & Positivo & Denatran \\
\hline & PPC & PIB per capita & Proporção & Positivo & IBGE \\
\hline
\end{tabular}

Fonte: Elaboração própria. 
As Figuras 1 e 2 apresentam o padrão de distribuição da razão entre a imigração de mão de obra qualificada e o total de trabalhadores qualificados do município de destino em 2001 e 2007.

Figura 1 - Razão entre a imigração de mão de obra qualificada e o total de trabalhadores qualificados para o ano de 2001

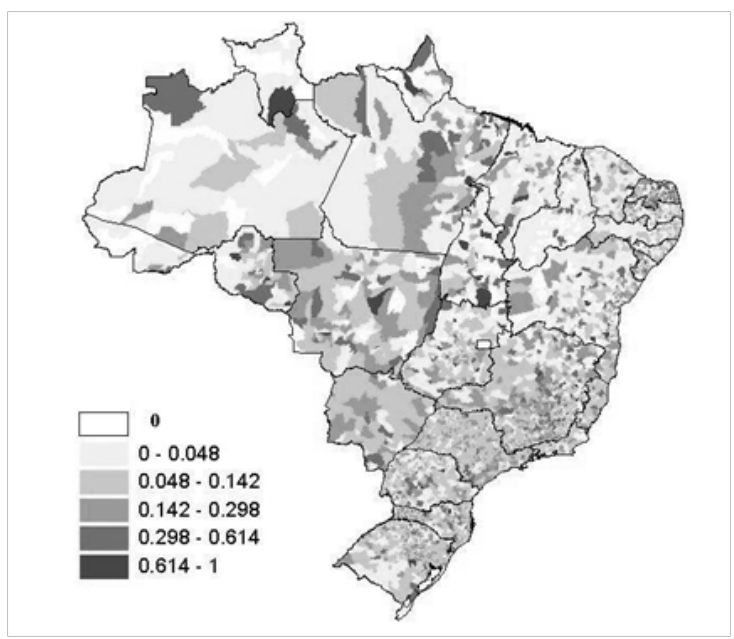

Fonte: Elaboração própria.

Figura 2 - Razão entre a imigração de mão de obra qualificada e o total de trabalhadores qualificados para o ano de 2007

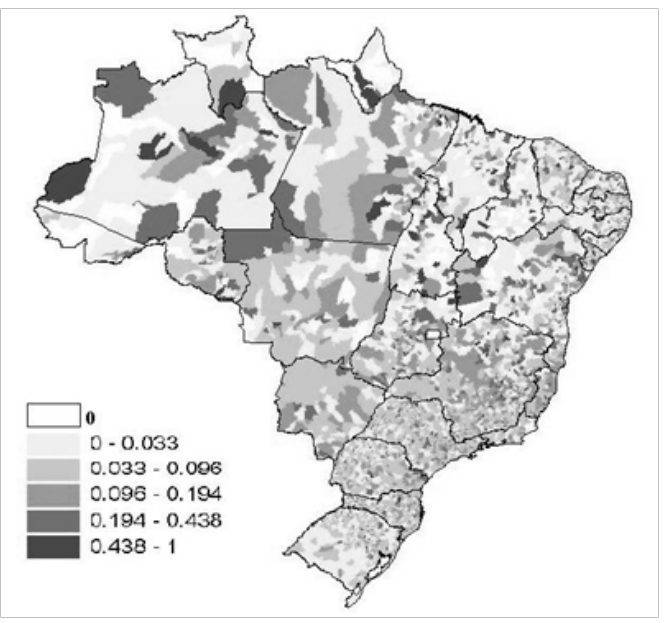

Fonte: Elaboração própria. 
Observa-se que no primeiro ano as regiões com municípios que mais recebem migrantes qualificados são a centro-oeste e a sudeste. A região nordeste apresenta pouca atratividade, mas o estado do Rio Grande do Norte se destaca como área receptora. ${ }^{4}$

No ano de 2007 o estado nordestino perde seu caráter receptor e os migrantes começam a se dirigir principalmente para os municípios do norte. Enquanto o sudeste permanece como importante destino desses trabalhadores, o sul perde sua função catalisadora. Cabe ressaltar, entretanto, que a média de mão de obra migrante qualificada em relação ao total de trabalhadores é maior no ano de 2001 do que em 2007.

A partir daí, para a variável dependente $\left(M Q P_{i}^{t}\right)$, foi checada a presença de autocorrelação espacial em nível global por intermédio das estatísticas I de Moran e $c$ de Geary. Os resultados são apresentados na Tabela 1.

Tabela 1 - Estatísticas globais de autocorrelação espacial para a variável $M Q P$

\begin{tabular}{c|cccccc}
\hline Ano & Indicador & Coeficiente & Média & $\begin{array}{c}\text { Desvio } \\
\text { padrão }\end{array}$ & Z-valor & P-valor \\
\hline \multirow{2}{*}{2001} & I de Moran & 0.0526 & 0.0000 & 0.0191 & 2.7721 & 0.0056 \\
& c de Geary & 0.9744 & 1.0000 & 0.0207 & -1.2406 & 0.2147 \\
& I de Moran & 0.0623 & 0.0000 & 0.0191 & 3.2801 & 0.0010 \\
& c de Geary & 0.9767 & 1.0000 & 0.0207 & -1.1267 & 0.2599 \\
\hline
\end{tabular}

Fonte: Elaboração própria.

A partir dos resultados pode-se perceber que, como o I de Moran é significativo em 1\%, rejeita-se a hipótese de aleatoriedade espacial da variável MQP. Além disso, há evidências de autocorrelação espacial positiva, ou seja, municípios com proporção de migrantes qualificados sobre o total de qualificados acima da média (abaixo da média) são vizinhos de municípios com $M Q P$ também acima da média (abaixo da média). Dessa forma, pode-se afirmar que há uma similaridade entre a proporção de migrantes e a localização, indicando concentração dos fluxos migratórios.

$4 \quad$ Para o cálculo dos coeficientes de autocorrelação, primeiro deve-se definir a matriz de pesos espaciais $W$ que determinará a disposição espacial das observações. Assim, para determinar qual matriz melhor captura a autocorrelação espacial, as de contiguidade, torre e rainha, ou uma matriz binária com $k$ vizinhos mais próximos, utilizou-se o procedimento de Baumont (2004). A partir deste, deve-se estimar o I de Moran para os resíduos do modelo econométrico, usando $N$ matrizes de $k$ vizinhos mais próximos e as torre e rainha e, assim, escolhe-se a matriz que gerar o maior valor do I de Moran significativo estatisticamente. 


\subsection{Modelo Econométrico}

Um painel de dados convencional é formado por informações na forma de corte cruzado e de período de tempo. O modelo tradicional de painel pressupõe a independência das unidades de corte cruzado, mas como o presente estudo trabalha com unidades espaciais (municípios), é preciso controlar a possível presença de dependência espacial entre os municípios. Dessa forma, adotou-se a princípio o modelo de painel sem dependência espacial e, em seguida, após testar a presença de autocorrelação espacial a fim de controlá-la, aplicou-se um modelo de efeitos fixos com dependência espacial.

O modelo utilizado se baseia na relação teórica entre a variável dependente e as explicativas:

$$
M Q P_{i j}=f\left(H O M P_{j}, F R O P_{j}, B U S P_{j}, I S A U_{j}, I E D U_{j}, I E R_{j} D R_{i j}, P O P_{j}, P P C_{j}, G I N D_{j}\right)
$$

em que $i$ é a região de origem e jé a de destino. O modelo demonstra que o indivíduo qualificado irá migrar atraído pelas características do destino.

A fim de se testar os fatores de atração do município, primeiramente realizou-se a estimação via mínimos quadrados ordinários para dados agrupados (POLS), efeitos aleatórios e efeitos fixos. O POLS, entretanto, não apresenta controles para os efeitos das características não observáveis dos municípios, como cultura, por exemplo. Essas características, portanto, poderiam estar correlacionadas com as variáveis explicativas, o que viesaria os resultados.

Para verificar a existência de efeitos não observados, foi realizado o teste de Breusch-Pagan. Dessa forma, se rejeitar a hipótese nula, há correlação entre os efeitos e as variáveis independentes e, consequentemente, deve-se corrigir o problema. Para isso, estimam-se os modelos de efeitos fixos e de efeitos aleatórios.

A fim de definir o melhor modelo para efeitos não observados (efeitos aleatórios ou fixos), realizou-se o teste de Hausman. Esse teste compara a consistência dos estimadores dos modelos, verificando possíveis diferenças entre eles. Ele testa a hipótese nula de presença de efeitos aleatórios, ou seja, que as estimativas dos modelos diferem sistematicamente. Se rejeitá-la, o modelo mais recomendado é o de efeitos fixos, se não, o melhor modelo é o de efeitos aleatórios. No presente estudo, como apresentado em seção posterior, o melhor modelo é o de efeitos fixos.

Após a escolha do melhor modelo, checa-se se os resíduos da estimação estão correlacionados espacialmente. Para verificar a presença da autocorrelação espacial, calcula-se o teste de I de Moran para os resíduos das unidades de corte cruzado para cada ano.

A autocorrelação espacial mede a correlação de valores para uma variável individual, que são estritamente imputáveis à proximidade desses valores no espa- 
ço geográfico ou socioeconômico. Dependendo de sua fonte, as interdependências espaciais podem ser capturadas por meio de um termo de defasagem espacial da variável dependente, das variáveis explicativas e/ou do termo de erro.

Dessa forma, ao estimar modelos de efeitos fixos com dependência espacial, controla-se para os dois principais problemas que poderiam tornar os resultados não confiáveis, a saber, a presença de efeitos não observados e a autocorrelação espacial. A especificação desse modelo empírico-econométrico geral pode ser representada por:

$$
\begin{gathered}
y_{t}=\rho W_{1} y_{t}+X_{t} \beta+W_{1} X_{t} \gamma+\mu+u_{t}, \quad \text { para } i=1, \ldots, N \text { e } t=1, \ldots, T \\
u_{t}=\lambda W_{2} u_{t}+\varepsilon_{t} \quad, \text { sendo } \varepsilon \sim\left(0, \sigma^{2} \mathrm{I}\right)
\end{gathered}
$$

em que $y_{t}$ é o vetor de observações da variável dependente; $X_{t}$ é uma matriz $n x$ $k$ de observações das variáveis explicativas; $\beta$ é o vetor de coeficientes a serem estimados; $W_{1} y_{t}$ é o vetor da variável dependente defasada espacialmente; $W_{1} X_{t}$ é a matriz que representa a defasagem espacial das variáveis explicativas; $\mu$ é o vetor do intercepto que captura os efeitos fixos de cada unidade espacial; $u_{t}$ é o vetor do termo de erro autocorrelacionado; $\varepsilon_{t}$ é o vetor do termo de erro i.i.d. com média zero e variância $\sigma^{2} ; W_{1}$ e $W_{2}$ são matrizes de ponderação espacial; $\rho$ é o parâmetro de defasagem autorregressiva $(-1<\rho<1)$; $\lambda$ é o parâmetro autorregressivo espacial $(-1<\lambda<1)$; e $\gamma$ é um vetor de coeficientes das externalidades da regressão.

Impondo restrições aos parâmetros, estimam-se o modelo de erro autorregressivo espacial, ou, como também é chamado, o modelo SEM $(\rho=0, \gamma=0$ e $\lambda$ $\neq 0$ ), o modelo de defasagem espacial, ou SAR $(\lambda=0, \gamma=0$ e $\rho \neq 0$ ), o modelo regressivo cruzado espacial, ou SLX $(\lambda=0, \rho=0$ e $\gamma \neq 0)$, o modelo Durbin espacial, ou SDM $(\lambda=0, \gamma \neq 0$ e $\rho \neq 0$ ), e o modelo Durbin espacial do erro, ou SDEM $(\rho=0, \lambda \neq 0$ e $\gamma \neq 0)$.

\section{Resultados}

A Tabela 2 reporta os resultados das regressões para dados em painel, sem controle para autocorrelação espacial, MQO empilhado (pooled OLS), efeitos aleatórios e efeitos fixos. Depois de estimado por MQO empilhado, realizou-se o teste de Breusch-Pagan a fim de detectar possíveis efeitos não observados no modelo, rejeitando a hipótese nula. Dessa forma, tendo em vista os possiveis problemas relativos à endogeneidade das variáveis explicativas, por estarem correlacionadas com 
o termo erro que contém variáveis não observadas, nas regressões por efeitos fixos e aleatórios consideram-se os efeitos não observados, apontados pelo teste.

Observa-se que o modelo de MQO empilhado, em relação aos outros modelos, apresenta coeficientes com os mesmos sinais, com a exceção do coeficiente do tamanho da população, que tem seu sinal invertido nos dois últimos. Apesar disso, destaca-se uma mudança da significância estatística de algumas variáveis entre os três modelos.

Sendo assim, estimou-se o teste de Hausman, cuja hipótese nula corresponde a estimadores de efeitos aleatórios ou fixos consistentes, mas somente os de efeitos aleatórios sendo eficientes. A hipótese alternativa do teste afirma a existência de estimadores de efeitos fixos consistentes. O valor do teste aponta para a rejeição da hipótese nula de existência de efeitos aleatórios, e, assim, o estimador mais eficiente entre os dois é o de efeitos fixos.

No modelo de efeitos fixos todos os coeficientes das variáveis explicativas são significativas estatisticamente em $1 \%$, sendo que as variáveis diferencial de renda esperado pelo trabalhador, tamanho da população, grau de industrialização, quantidade de ônibus per capita e tamanho da população apresentam correlação positiva, enquanto que os índices Firjan, PIB per capita, homicídio per capita e frota de veículos per capita têm relação negativa. Destaca-se que o coeficiente da variável de população, apesar de apresentar significância estatística, possui coeficiente muito próximo a zero.

Tabela 2 - Regressões em painel sem controle para dependência espacial

\begin{tabular}{llll}
\hline & \multicolumn{4}{l}{ Variável dependente: MQP } \\
\hline POLS & EA & EF \\
\hline Constante & $0,2070 * * *$ & $0,2400 * *$ & $0,4048 * * *$ \\
\multirow{2}{*}{ DR } & $(0,0166)$ & $(0,0081)$ & $(0,0000)$ \\
& $0,0359 * * *$ & $0,0351 * * *$ & $0,0385 * * *$ \\
GIND & $(0,0091)$ & $(0,0077)$ & $(0,0000)$ \\
& $0,0312 * * *$ & $0,0464 * * *$ & $0,0353 * * *$ \\
ISAU & $(0,0100)$ & $(0,0087)$ & $(0,0000)$ \\
& $-0,123 * * *$ & $-0,1578 * * *$ & $-0,2230 * * *$ \\
IER & $(0,0214)$ & $(0,0169)$ & $(0,0000)$ \\
& $0,0481 * * *$ & 0,0479 & $-0,0105 * * *$ \\
IEDU & $(0,0129)$ & $(0,0558)$ & $(0,0000)$ \\
& $-0,0608 * * *$ & $-0,0997 * * *$ & $-0,2354 * * *$ \\
& $(0,0152)$ & $(0,0167)$ & $(0,0000)$ \\
\hline
\end{tabular}


conclusão...

\begin{tabular}{llll}
\hline \multicolumn{4}{l}{ Variável dependente: MQP } \\
\hline PPC & POLS & EA & EF \\
& $0,00155^{* * *}$ & 0,0017 & $-0,0009^{* * *}$ \\
HOMP & $(0,0004)$ & $(0,0013)$ & $(0,0000)$ \\
& $-5,250$ & $-15,333$ & $-4,4005^{* * *}$ \\
FROP & $(8,2037)$ & $(11,7217)$ & $(0,0000)$ \\
& $-0,00119$ & $-0,0010^{* *}$ & $-0,0044^{* * *}$ \\
BUSP & $(0,0020)$ & $(0,0005)$ & $(0,0000)$ \\
& 0,234 & $0,1839 * * *$ & $0,3091^{* * *}$ \\
POP & $(0,2012)$ & $-0,0309$ & $(0,0000)$ \\
& $-0,0000^{* * *}$ & $0,0000^{* * *}$ & $0,0000^{* * *}$ \\
\hline Teste de Breusch e & $(0,0000)$ & $(0,0000)$ & $(0,0000)$ \\
Pagan & $\operatorname{chi}^{2}(1)=127,81$ & & Prob $>$ chi $^{2}=0,0000$ \\
Teste de Hausman & $\operatorname{chi}^{2}(8)=116,12$ & & \\
R & 0,0548 & 0,0953 & 0,1117 \\
\hline
\end{tabular}

Fonte: Elaboração própria.

Nota: *** Significativo em 1\%; ** em 5\%; * em 10\%. Erro-padrão entre parênteses.

A partir dos resíduos do modelo estimado por efeitos fixos apresentado na Tabela 2 , eles foram checados para a presença de autocorrelação espacial. Por meio da estatística I de Moran, foi detectada a dependência espacial nos anos de 2001 e 2007 nos resíduos do modelo de efeitos fixos, uma vez que a estatística se mostrou significativa em $1 \%$ para ambos os anos.

Sendo assim, a fim de controlar a autocorrelação espacial e os efeitos não observados, estimaram-se os modelos em painel de dados com correção para dependência SAR, SLX, SDM, SDEM e SEM. Para correção da dependência espacial, deve-se escolher como melhor modelo aquele em que os resíduos não apresentarem autocorrelação e o modelo apresentar o melhor ajuste apontado por um valor do critério de informação, tal como o critério de Akaike.

A Tabela 3 apresenta o I de Moran para os resíduos dos modelos espaciais de efeitos fixos. Observa-se, portanto, que os modelos que a 1\% de significância controlam para a dependência espacial, já que apresentam o I de Moran não significativo, são SEM, SDEM e SDM. Por fim, conclui-se que o melhor modelo, portanto, é o SDEM uma vez que possui o menor Akaike e a maior log-verossimilhança $(10409,6)$. 
Tabela 3 - I de Moran para os resíduos dos modelos espaciais de efeitos fixos

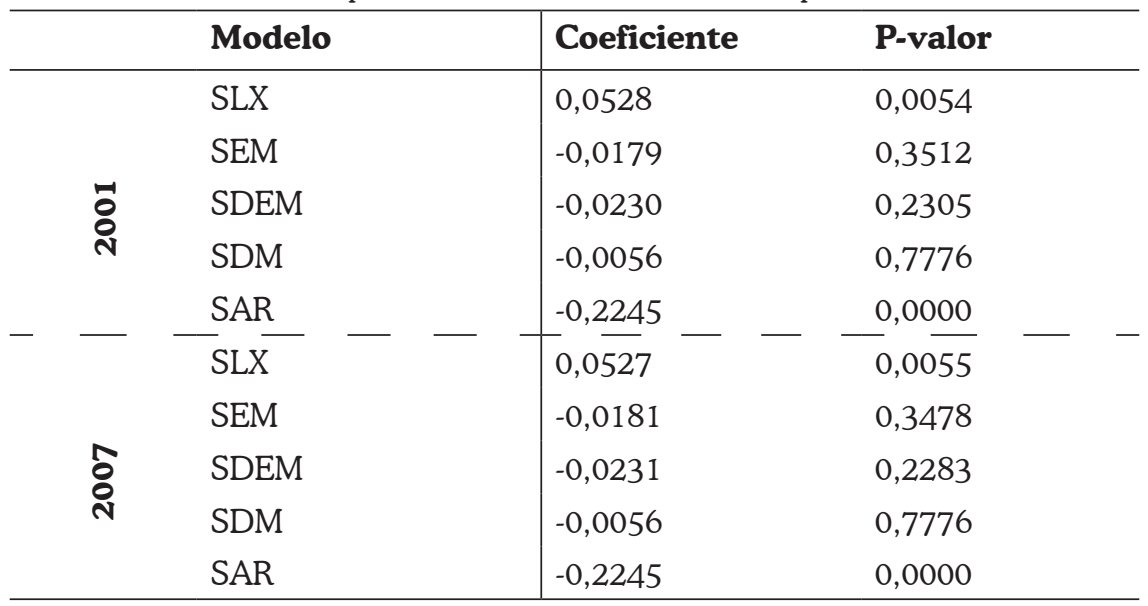

Fonte: Elaboração própria.

Dessa forma, apresentam-se na Tabela 4 os resultados do modelo SDEM com controle para efeitos fixos. Destaca-se que todas as variáveis e suas defasagens espaciais se mostram significativas em $1 \%$. Em relação ao modelo de efeitos fixos sem controle para dependência espacial, observa-se uma mudança de sinal de alguns coeficientes, comprovando-se o viesamento dos coeficientes estimados do primeiro modelo pela falta de controle da autocorrelação espacial.

Destaca-se uma relação positiva entre o diferencial de renda esperada e a proporção da mão de obra qualificada migrante, sendo que o seu coeficiente é muito superior ao mostrado anteriormente. Tal resultado era esperado, uma vez que a decisão de migrar é muito relacionada a variáveis pecuniárias. De forma semelhante, as variáveis PIB per capita, tamanho da população e grau de industrialização são relacionadas positivamente com a variável de interesse. Apesar da significância estatística da variável, observa-se um coeficiente muito próximo a zero. Esses sinais dos coeficientes mostram-se de acordo com a literatura.

As variáveis de amenidades, ônibus per capita, homicídio per capita e frota de veículos per capita apresentam os resultados esperados, tendo a primeira correlação positiva e as duas últimas, negativa. Dessa forma, pode-se afirmar que os municípios com um melhor transporte público, um menor índice de violência e com um congestionamento menor atraem uma maior proporção de migrantes qualificados. As variáveis do índice Firjan de educação e de saúde apresentam relação negativa, ao passo que as de emprego e renda revelam-se positivamente associadas com a variável dependente. Essa correlação pode ser justificada pelo fato de os migrantes qualificados migrarem principalmente em busca de melhores condições de trabalho. 
Ao analisar o efeito indireto, ou seja, as variáveis defasadas espacialmente, observa-se que o valor da população, o índice Firjan de educação, renda esperada, grau de industrialização, número de ônibus e número de veículos per capita das regiões vizinhas contribuem positivamente para a proporção de migrantes qualificados sobre o total de trabalhadores com ensino superior completo da região. Já as variáveis Firjan de saúde e emprego e renda, homicídio e PIB per capita contribuem negativamente.

Em relação à renda esperada, pode-se justificar tal relação pelo fato de uma maior renda dos municípios vizinhos implicar uma maior atratividade da região como um todo, aumentando inclusive a imigração do próprio município. A mesma interpretação pode ser aplicada às variáveis de grau de industrialização, tamanho da população e educação básica, que indicarão um maior desenvolvimento de toda a região. As variáveis representando transporte público e frota de veículos, por apresentarem sinal positivo, indicam que uma maior congestão dos vizinhos aumentaria a imigração do município. Isso pode ocorrer pelo fato de que os trabalhadores, apesar de almejarem o desenvolvimento da região, buscam fugir das amenidades negativas, refugiando-se em municípios próximos.

Tabela 4 - Regressões em painel com controle para dependência espacial

\begin{tabular}{|c|c|}
\hline \multirow{2}{*}{\multicolumn{2}{|c|}{$\begin{array}{c}\text { Variável dependente: } \mathrm{MQF} \\
\text { SDEM por efeitos fixos }\end{array}$}} \\
\hline & \\
\hline \multirow[t]{2}{*}{ Constante } & $0,0855^{* * *}$ \\
\hline & $(0,0000)$ \\
\hline \multirow[t]{2}{*}{$\mathrm{DR}$} & $0,0429 * * *$ \\
\hline & $(0,0000)$ \\
\hline \multirow[t]{2}{*}{ GIND } & $0,0254 * * *$ \\
\hline & $(0,0000)$ \\
\hline \multirow[t]{2}{*}{ IEDU } & $-0,0303 * * *$ \\
\hline & $(0,0000)$ \\
\hline \multirow[t]{2}{*}{ ISAU } & $-0,0151 * * *$ \\
\hline & $(0,0000)$ \\
\hline \multirow[t]{2}{*}{ IER } & $0,0641 * * *$ \\
\hline & $(0,0000)$ \\
\hline \multirow[t]{2}{*}{ POP } & $0,0002 * * *$ \\
\hline & $(0,0000)$ \\
\hline \multirow[t]{2}{*}{ PPC } & $0,0005 * *$ \\
\hline & $(0,0000)$ \\
\hline
\end{tabular}


conclusão...

\begin{tabular}{|c|c|}
\hline \multirow{2}{*}{\multicolumn{2}{|c|}{$\begin{array}{c}\text { Variável dependente: MQP } \\
\text { SDEM por efeitos fixos }\end{array}$}} \\
\hline & \\
\hline \multirow[t]{2}{*}{ BUSP } & $0,7353 * * *$ \\
\hline & $(0,0000)$ \\
\hline \multirow[t]{2}{*}{ HOMP } & $-22,0640 * * *$ \\
\hline & $(0,0000)$ \\
\hline \multirow[t]{2}{*}{ FROP } & $-0,0075 * * *$ \\
\hline & $(0,0000)$ \\
\hline \multirow{2}{*}{ WDR } & $1,0000 * * *$ \\
\hline & $(0,0000)$ \\
\hline \multirow[t]{2}{*}{ WGIND } & $0,0049 * * *$ \\
\hline & $(0,0000)$ \\
\hline \multirow[t]{2}{*}{ WIEDU } & $0,0106 * * *$ \\
\hline & $(0,0000)$ \\
\hline \multirow[t]{2}{*}{ WISAU } & $-0,0040 * * *$ \\
\hline & $(0,0000)$ \\
\hline \multirow[t]{2}{*}{ WIER } & $-0,0030 * * *$ \\
\hline & $(0,0000)$ \\
\hline \multirow[t]{2}{*}{ WPOP } & $0,0000 * * *$ \\
\hline & $(0,0000)$ \\
\hline \multirow[t]{2}{*}{ WPPC } & $-0,0005 * * *$ \\
\hline & $(0,0000)$ \\
\hline \multirow[t]{2}{*}{ WBUSP } & $0,0056 * * *$ \\
\hline & $(0,0000)$ \\
\hline \multirow[t]{2}{*}{ WHOMP } & $-0,2377 * * *$ \\
\hline & $(0,0000)$ \\
\hline \multirow[t]{2}{*}{ WFROP } & $0,0012 * * *$ \\
\hline & $(0,0000)$ \\
\hline & $R^{2}=0,5915$ \\
\hline
\end{tabular}

Fonte: Elaboração própria.

Nota: ** * Significativo em 1\%; * em 5\%; * em 10\%. Erro-padrão entre parênteses. 
As variáveis com coeficiente negativo indicam que um maior desenvolvimento de um município vizinho diminuiria a imigração de outro. Tal fato está relacionado à maior atração exercida pelo vizinho sobre o imigrante que deixaria de entrar no município a fim de buscar melhores empregos, saúde básica e crescimento municipal (indicados pelo índice Firjan de emprego e renda, saúde e PIB per capita, respectivamente) no vizinho.

\section{Considerações Finais}

Altos níveis de migração geográfica de pessoas qualificadas asseguram o nível de inovação e de desenvolvimento econômico da região (GRAVERSEN; FRIIS-JENSEN, 2001), sendo a migração um dos principais meios pelos quais ocorrem os transbordamentos de conhecimento. Por intermédio da migração, regiões podem ter acesso a conhecimentos que por outro meio não teriam.

Ao avaliar a variável de interesse, a saber, a proporção de trabalhadores migrantes qualificados sobre o total da mão de obra qualificada, rejeita-se a hipótese de aleatoriedade espacial da variável. Assim, comparando com o modelo de efeitos fixos sem controle para dependência espacial, nota-se uma reversão do sinal de alguns coeficientes, podendo-se, assim, confirmar o viesamento das estimativas do primeiro modelo pela falta de controle da autocorrelação espacial.

Observa-se uma relação positiva entre o diferencial de renda esperada e a proporção da mão de obra qualificada migrante. Essa correlação se repete para as variáveis PIB per capita, tamanho da população e grau de industrialização, indicando que os trabalhadores qualificados migrantes são atraídos pelas regiões mais desenvolvidas. Além disso, essa mão de obra tem preferência por cidades com um melhor transporte público, um menor índice de violência e com um congestionamento menor.

Por fim, constata-se que o tamanho da população, da renda esperada, do grau de industrialização, do número de ônibus e do número de veículos das regiões vizinhas contribui positivamente para a entrada de migrantes no município. Já as variáveis de homicídio e PIB per capita contribuem negativamente. Desse modo, constata-se que, apesar de os migrantes qualificados serem atraídos por regiões mais desenvolvidas, o desenvolvimento superior de um município vizinho faz com que esses trabalhadores se dirijam para ele. Com isso, há uma concorrência na atração desses trabalhadores entre os municípios e uma atração conjunta para a região.

De forma semelhante, observa-se uma fuga dos municípios com congestão, sendo que os trabalhadores fogem para os vizinhos. Cabe destacar, portanto, que as cidades que desejarem atrair esse tipo de mão de obra devem investir na 
melhoria de suas amenidades e em desenvolvimento urbano, além da melhoria de sua renda esperada.

\section{Referências}

ADAMS JR, R. H.; PAGE, J. Do international migration and remittances reduce poverty in developing countries? World Development, v. 33, n. 10, p. 1645-1669, 2005.

ALMEIDA, E. Econometria espacial aplicada. Campinas: Alínea Editora, 2012.

ANDRIENKO, Y.; GURIEV, S. Determinants of interregional mobility in Russia. Economics of Transition, v. 12, n.1, p.. 1-27, 2004.

BAENINGER, R. São Paulo e suas migrações no final do século 20. São Paulo em Perspectiva, v. 19, n. 3, p. 84-96, set. 2005.

BORJAS, G. J. Labor mobility. In: . Labor economics. Singapura: McGraw-Hill, 1996. p. 279-317.

. Self-selection and the earnings of immigrants. American Economic Review, v. 77, n. 4, p. 531-553, Sept. 1987.

. The labor market impact of high-skill immigration. American Economic Review, v. 95, n. 2, p. 56-60, 2005.

BOVER, O.; ARELLANO, M. Learning about migration decisions from de migrants: using complementary dataset to model intra-regional migrations in Spain. Population Economics, v. 15 , n. 2 , p. $357-380,2002$.

BRAGA, F. G. Migração interna e urbanização no brasil contemporâneo: um estudo da rede de localidades centrais do Brasil (1980/2000). In: ENCONTRO NACIONAL DE ESTUDOS POPULACIONAIS, 15., 2006, Caxambu. Anais... Caxambu: Associação Brasileira de Estudos Populacionais, 2006.

CAMERON, A. C.; TRIVEDI, P. K. Microeconometrics: methods and applications. Cambridge, MA: Cambridge University Press, 2005.

CEBULA, R. J.; ALEXANDER, G. M. Determinants of net interstate migration, 2000-2004. The journal of Regional Analysis and Policy, v. 2, n. 36, p. 116-123, 2006.

CEBULA, R. J. Internal migration determinants:recent evidence. International Advances in Economic Research, v. 11, n. 3, p. 267-274, 2005.

CHEN, Y.; ROSENTHAL, S. S. Local amenities and life-cycle migration: do people move for jobs or fun? Journal of Urban Economics, v. 64, n. 3, p. 519-537, 2008.

CHISWICK, B. R. Are immigrants favorably self-selected? An economic analysis. In: BRETTELL, C. D.; HOLLIFIELD, J. F. Migration theory: talking across the disciplines. Nova York: Routledge, 1999. p.52-75. 
DAHL, M.; SORENSON, O. The migration of technical workers, Journal of Urban Economics, v. 67, n. 1, p. 33-45, 2010.

DAHL, M. S. Embodied knowledge diffusion, labor mobility and regional dynamics: do social factors limit the development potential of regions? In: DRUID SUMMER CONFERENCE, 2004, Elsinore. Proceedings... Marienlyst, Dinamarca: VBN, 2004.

DEBELLE, G; VICKERY, J. Labour Market Adjustment: Evidence on Interstate LabourMobility. Australian Economic Review, v. 32, n. 3, p. 249-263, 1999.

FERREIRA, R. N.; MATOS, R. Migração de trabalhadores no mercado formal brasileiro entre 1995 e 2003 e as tendências da reestruturação territorial. In: ENCONTRO NACIONAL DE ESTUDOS POPULACIONAIS, 14, 2004, Caxambu. Anais... Belo Horizonte: ABEP, 2006.

FIRJAN. IFDM: Índice Firjan de Desenvolvimento Regional. Disponível em: http://www.firjan.org.br/ifdm/downloads/

FREGUGLIA, R. S.; MENEZES-FILHO, N. A. Inter-regional and inter-industry wage differentials with individual heterogeneity: estimates using Brazilian data. In: MIDWEST ECONOMICS ASSOCIATION CONFERENCE, 2007. Proceedings... Minneapolis, MN: Midwest Economics Association, 2007.

GIBSON, J.; MCKENZIE, D. The microeconomic determinants of emigration and return migration of the best and brightest: evidence from the Pacific. Journal of Development Economics, v. 95, n. 1, p. 18-29, 2011.

GOLGHER, A. B. As cidades e a classe criativa no Brasil: diferenças espaciais na distribuição de indivíduos qualificados nos municípios brasileiros. Revista Brasileira de Estudos da População, v. 25, n. 1, p. 109-129, 2008.

GOLGHER, A. ; ROSA, C. H. ; ARAUJO JR, A. F. The determinants of migration in Brazil. In: Proceedings of the 33th Brazilian Economics Meeting. Niterói: Associação Nacional dos Centros de Pós-graduação em Economia. 2005. p. 1-20.

GRAVERSEN, E. K.; FRIIS-JENSEN, K. Job mobility implications of the human resources in science and technology definition: illustrated by empirical numbers from register data. In: ORGANISATION FOR ECONOMIC CO-OPERATION AND DEVELOPMENT. (Org.). Innovative people: mobility of skilled personnel in national innovation systems. Paris: OECD, 2001. p. 45-58.

IPEA. Ipeadata: dados macroeconômicos e regionais. Disponível em: http://www.ipeadata.gov.br/

JACKMAN, R.; SAVOURI, S. Regional migration in Britain: an analysis of gross flows using NHS central register data. The Economic Journal, v. 102, n. 415, p. 1433-1450, nov. 1992.

JAEGER, D. et al. Direct evidence on risk attitudes and migration. Review of Economics and Statistics, v. 92, n. 3, p. 689-692, 2010.

JENSEN, T.; DELLER, S. Spatial modeling of the migration of older people with a focus on amenities. The Review of Regional Studies, v. 37, n. 3, p. 303-343, 2007. 
JUSTO, W. R.; SILVEIRA NETO, R. M. Migração inter-regional no Brasil: evidências a partir de um modelo espacial. Economia, Brasília, DF, v. 7, n. 1, p. 163-187, jan./jul. 2006.

KULU, H.; BILLARI, F. C. Multilevel analysis of internal migration in a transitional country: the case of Estonia. Regional Studies, v. 38, n. 6, p. 697-696, Aug. 2004.

LUCAS, Robert EB. The effects of proximity and transportation on developing country population migrations. Journal of Economic Geography, v. 1, n. 3, p. 323-339, 2001.

MATA, D. et al. Quais características das cidades determinam a atração de migrantes qualificados? In: ENCONTRO REGIONAL DE ECONOMIA, 12., 2007, Fortaleza. Anais... Fortaleza: Banco do Nordeste, 2007.

MATA, D. et al. Migração, qualificação e desempenho das cidades brasileiras. In: CARVALHO, A. X. Y. et al. (Org.). Dinâmica dos municípios. Brasília, DF: Ipea, 2008. p. 289-322.

MATOS, R. E. S.; BRAGA, F. G. Migração e rede urbana: procedências e inserção ocupacional. In: ENCONTRO NACIONAL DE ESTUDOS POPULACIONAIS, 13., 2002, Ouro Preto. Anais... Ouro Preto: Associação Brasileira de Estudos Populacionais, 2002.

MENEZES, T.; FERREIRA JÚNIOR, D. Migração e convergência de renda. In: VIII Encontro Regional de Economia, 8., 2003, Fortaleza. Anais... Fortaleza: Anpec, 2003.

MTE. Ministério do trabalho e emprego: dados e estatísticas. Disponível em: http://portal.mte.gov.br/rais/estatisticas.htm

MUKKALA, K. Knowledge spillovers: mobility of highly educated workers within high technology sector in Finland. In: CONGRESS OF THE EUROPEAN REGIONAL SCIENCE ASSOCIATION, 45., 2005, Amsterdam, Holanda. Proceedings... Amsterdam, Holland: ERSA, 2005.

NAKOSTEEN, R. A.; WESTERLUND, O. The effects of regional migration on gross income of labour in Sweden. Regional Science, v. 83, n. 3, p. 581-595, 2004.

OLIVEIRA, K. F.; JANNUZI, P. M. Motivos para migração no Brasil e retorno ao nordeste: padrões etários, por sexo e origem/destino. São Paulo em Perspectiva, v. 19, n. 4, p. 134-143, dez. 2005.

PEKKALA, S. Migration flows in Finland: regional differences in migration determinants and migrant types. International Regional Science Review, v. 26, n. 4, p. 466-482, Oct. 2003.

PISSARIDES, C.; WADSWORTH, J. Unemployment and the Inter-Regional Mobility of Labour. The Economic Journal, v. 99, n. 397, p. 739-755, 1989.

QUEIROZ, B. L.; GOLGHER, A. B. Human capital differentials across municipalities and states in Brazil. Belo Horizonte: UFMG/Cedeplar, 2008. (Texto para discussão, n. 330).

QUEIROZ, B. L.; CÉSAR, C. C. Dinâmica econômica, mercado de trabalho e diferenciais de salários nos estados brasileiros. In: ENCONTRO NACIONAL DE ESTUDOS POPULACIONAIS, 12., 2000, Belo Horizonte. Anais... Belo horizonte: ABEP, 2000.

RIGOTTI, J. I. R. Geography of population flow according to the migrants educational level. Estudos Avançados, São Paulo, v. 20, n. 57, p. 237-254, 2006. 
ROMER, P. Endogenous technological change. Journal of Political Economy, v. 98, n. 5, p. 71-102, 1990.

SACHSIDA, A. et al. Perfil do migrante brasileiro. Rio de Janeiro: Ipea, 2009. (Texto para Discussão, n. 1410).

SACHSIDA, A.; CAETANO, M. A.; ALBUQUERQUE, P. Distribuição de renda, transferências federais e imigração: um estudo de dados em painel para as Unidades da Federação do Brasil. Rio de Janeiro: IPEA, 2010. (Texto de Discussão, n. 1471).

SANTOS JUNIOR, E. R.; MENEZES FILHO, N. A.; FERREIRA, P. C. Migração, seleção e diferenças regionais de renda no Brasil. Pesquisa e Planejamento Econômico, Rio de Janeiro, v. 35, n. 3, p. 299-332, 2005.

SJAASTAD, L. A. The costs and returns of human migration. Journal of Political Economy, v. 70, n. 5, p. 80-93, Oct. 1962.

STAMBOL, L. S. Urban and regional labour mobility performance in Norway. In: CONGRESS OF THE EUROPEAN REGIONAL SCIENCE ASSOCIATION, 43. 2003, Jyväskylä, Finland. Proceedings... Jyväskylä, Finland: ERSA, 2003.

WOOLDRIDGE, J. M. Econometric analysis of cross section and panel data. London: MIT Press, 2002.

ZIMMERMANN, Klaus F. European labour mobility: challenges and potentials. De Economist, v. 153, n. 4, p. 425-450, 2005.

Recebido em: 13/09/2011. Aceito em: 11/04/2014. 